\title{
Evaluation of Cocos nucifera and Sesamum indicum in comparison with Chlorhexidine on gingivitis - A double blind Clinico-Immunological study
}

\author{
Research Article
}

\section{Ranjini Chandraprakash1, Ravindra Shivamurthy', Vivekananda Maranahally Rangaraju ${ }^{3}$, Harsha Mysore Babu ${ }^{4 *}$}

1. Clinical Practitioner, Mahadevpura main road, Near Jalpuri, Mysuru, 2. Principal, JSS Dental College and Hospital, SS Nagar, Mysuru, 3. Professor, 4. Professor and Head, Department of Periodontics, Sri Hasanamba Dental College and Hospital, Vidyanagar, Hassan.

\begin{abstract}
Background: Myriad of synthetic products has been used in chemical plaque control. There is a constant search for cost effective herbal products with minimal adverse effect to substitute synthetic compounds. The objective of this study was to evaluate the anti-inflammatory properties of Cocos nucifera and Sesamum indicum and compare their effect with commercially available chlorhexidine on gingivitis. Methods: In this double blind, randomized, control clinical trial, a total of 45 samples from patients aged between 18 to 35 years reporting to the institution, diagnosed with gingivitis were selected and randomly divided into Group-A (Scaling + Cocos Nucifera mouthwash), Group-B (Scaling + Sesamum Indicum mouthwash) and Group C (Scaling + Chlorhexidine mouthwash). Clinical (Plaque index, Gingival index and Sulcus bleeding index), and Immunological (Interleukin-6) parameters were assessed at baseline and $45^{\text {th }}$ day following scaling. Saliva samples were collected and stored at $-20^{\circ} \mathrm{C}$ till they were subjected to enzyme-linked immunosorbent assay (ELISA) analysis. Inferential statistics done were analysis of variance, paired t test, post hock Scheffe test and Chi- square test by using SPSS software (22.0). Results: In Clinical parameters, group B $(\mathrm{p}<0.001)$ showed statistical significant reduction compared to group A and group C. In Immunological parameter group A $(\mathrm{p}<0.001)$ showed statistical significant reduction in Interleukin-6 compared to group B and group C ( $\mathrm{p}=0.126 \& \mathrm{p}=0.196$ respectively). Conclusion: Cocos nucifera and Sesamum indicum mouth washes effectively decreased plaque formation and could be used as an adjunct to scaling in treating plaque induced gingivitis.
\end{abstract}

Key Words: Cocos Nucifera, Chlorhexidine, Chemical plaque control, Gingivitis, Interluekin-6, Sesamum Indicum.

\section{Introduction}

Periodontal disease is one of the commonest diseases of humankind affecting 20-50\% of global population including all age groups. Therefore its prevention is of utmost importance. Along with mechanical methods of plaque control, various antimicrobials have been used as adjunctive measures (1). Chlorhexidine is considered a gold standard against which efficacy of other antiplaque agents are compared (2). Although having high substantivity, the use of chlorhexidine for prolonged durations is restricted due to its side effects such as extrinsically staining the teeth, altered taste sensation, mucosal erosions and a general burning sensation of tongue (3).

\section{* Corresponding Author:}

\section{Harsha Mysore Babu}

Professor and Head, Department of Periodontics,

Sri Hasanamba Dental College and Hospital,

Vidyanagar,

Hassan - 573202. India

Email Id: harshamb@yahoo.com
There is a constant search for cost effective herbal products with minimal adverse effect to substitute synthetic compounds. Coconut oil is derived from the nuts of the plant Cocos nucifera. The Lauric acid component has been shown to have antiinflammatory, antimicrobial, antiviral, and anti-fungal activities (4). In vitro, its sucrose monolaurate content has shown to prevent formation of dental plaque (5).

Sesame oil, derived from Sesamum indicum, is known for its antimicrobial effects has shown considerable reduction in plaque formation, when used in the form of oil pulling (6).

There is paucity in the literature comparing the efficacy of Cocos nucifera and Sesamum indicum on periodontal diseases. Hence this study was conducted evaluate and compare the antiplaque and antiinflammatory properties of Cocos nucifera and Sesamum indicum with the gold standard antimicrobial agent chlorhexidine in the treatment of plaque induced gingivitis.

\section{Materials and methods}

A controlled, double blinded randomized clinical and immunological study was conducted among 
the out-patients visiting the Department of Periodontics, of the institution from September 2016 to March 2017. The study design was approved by the Ethical Committee of the institution. Subjects aged between 18 to 35 years, diagnosed with gingivitis, not on any antibiotic or anti-inflammatory drug in the preceding 6 months and have not undergone dental treatment for the past three months were selected. Subjects who were smokers, pregnant or lactating females, with any systemic diseases were excluded from the study.

Method of preparation of Cocos nucifera and Sesamum indicum mouthwash: Both Cocos nucifera and Sesamum indicum mouthwash were prepared by the pharmacist of Ayurvedic College.

Cocos nucifera (Coconut oil) mouthwash: The micro-emulsion mouthwash containing essential oil of $5 \%$ concentration coconut oil, tween 80 , glycerine, peppermint oil, sodium benzoate and water was prepared. Coconut oil, peppermint oil and tween 80 were mixed to obtain oil phase. Sodium benzoate solution, Glycerine, and water were mixed to obtain water phase. The water phase was added to oil phase and immediately mixed homogenously. An adequate amount of water was added to reach final volume. The ingredients to make $100 \mathrm{ml}$ of mouth wash are as follows: Coconut oil $(20 \mathrm{ml})+$ Peppermint oil $(1 \mathrm{ml})+$ Tween $80(2 \mathrm{ml})+$ Glycerine $(4.8 \mathrm{ml})+$ Sodium benzoate $(1 \mathrm{~g})+$ Water $(71.2 \mathrm{ml})$. The formulation was stored in amber coloured glass bottles.

Sesamum indicum (Sesame oil) mouth wash: The micro-emulsion mouthwash containing essential oil of $10 \%$ concentration sesame oil, tween 80 , glycerin, peppermint oil, sodium benzoate and water was prepared. Sesame oil, peppermint oil and tween 80 were mixed to obtain oil phase. Glycerine, sodium benzoate solution, water was mixed to obtain water phase. The water phase was added to oil phase and immediately mixed to homogenous. An adequate amount of water was added to reach final volume. The ingredients to make $100 \mathrm{ml}$ of mouth wash are as follows: Sesame oil $(20 \mathrm{ml})+$ Peppermint oil $(1 \mathrm{ml})+$ Tween $80(2 \mathrm{ml})+$ Glycerine $(4.8 \mathrm{ml})+$ Sodium benzoate $(1 \mathrm{~g})+$ Water $(71.2 \mathrm{ml})$. The formula was stored in glass bottles. The formulation was kept in amber coloured glass bottles.

Clinical procedure: The purpose of the study and design were explained, and a written consent was obtained from all the participants. The sample size was estimated by considering $\alpha$ error probability of 0.05 and power of the study was $80 \%$, hence, sample size calculated was 15 patients per group. Forty-five individuals meeting the inclusion criteria were randomly assigned to one of the three groups using chit method as follows:

- Group A: (15 subjects) - Scaling + Cocos nucifera mouthwash

- Group B: (15 subjects) - Scaling + Sesamum indicum mouthwash

- Group C: (15 subjects) - Scaling + 0.2\% Chlorhexidine mouthwash

The control $(0.2 \%$ chlorhexidine $)$ and freshly prepared both the test agents were dispensed in amber coloured bottles with a code by a non-participant.
Following scaling, the subjects in each group were given respective mouth washes and were advised to rinse the mouth $(10 \mathrm{ml})$ twice daily for 2-3 minutes following their oral hygiene routine for 45 days.

The special case history proforma was designed to record the findings included in the study. The clinical parameters: Plaque Index (PI) (7), Gingival Index (GI) (8) and Sulcus bleeding index (SBI) (9) were recorded at baseline and at $45^{\text {th }}$ day after using the prescribed mouthwashes. An examiner who was blinded towards the participant allocation recorded all clinical parameters.

Saliva samples were collected at baseline and $45^{\text {th }}$ day before clinical examination of the subjects and subjected to immunological assay. Each subject was advised to collect saliva in their mouth for about 5 minutes and spit into a sterile plastic glass. Then saliva was transferred into a labeled sterile plastic tube with syringe and stored in at $-20{ }^{\circ} \mathrm{C}$ till the assay procedure. The samples were transported to the laboratory in a cold pack for assessment of Interleukin (IL)-6 using Human ELISA kit (GENXBIOC), GXBH141938).

The study design is represented in consort chart (Figure 1)

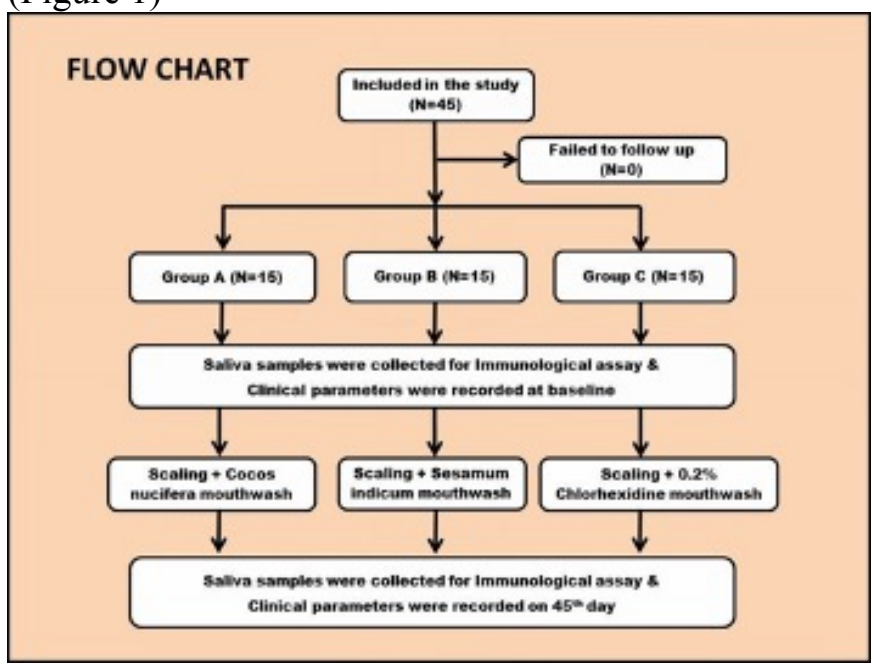

\section{Statistical analysis}

Data collection was subjected to statistical analysis by applying ANOVA, paired t test, post hock Scheffe test and Chi- square test by using SPSS software (22.0) to assess the objectives of the study. Probability value $<0.05$ was considered as statistically significant.

\section{Results}

In the present double blinded randomized controlled clinico-immunological study, the selected 45 subjects aged between 18 to 35 years were randomly assigned into three groups.

The mean age for Group A, Group B and Group C were 26.1, 29.6 and 28.6 respectively.

The mean PI score at baseline for Group A, Group B and Group C were $0.915 \pm 0.303,0.917 \pm$ 0.088 , and $0.737 \pm 0.185$ respectively. There was no statistical significant difference in mean PI score between 
group $\mathrm{A}$, group $\mathrm{B} \&$ group $\mathrm{C}$ at baseline at $5 \%$ significant level $(p=0.037)$. The mean PI score on $45^{\text {th }}$ day for Group A, Group B and Group C were $0.100 \pm 0.041,0.077 \pm$ 0.031 and $0.074 \pm 0.030$ respectively. There was no statistical significant difference in mean PI score between Group A, Group B \& Group C at 45 days at 5\% significance level $(p=0.095)$ (Table 1$)$.

The PI score reduction from baseline to 45 days was found to be $0.815 \pm 0.269$ in Group A, $0.839 \pm 0.086$ in Group B and $0.663 \pm 0.191$ in Group C. One way ANOVA was used to check the PI score reduction among the groups. It showed a statistically significant difference in mean PI reduction. (Table 2) A pair wise comparison was done using Scheffe post hoc test, there was no statistical significant difference in PI score reduction in sub groups (Group A, Group B and Group C).

The mean GI score at baseline for Group A, Group B and Group C were $0.571 \pm 0.238,0.865 \pm 0.164$ and $0.552 \pm 0.241$ respectively. There was a statistical significant difference in mean GI score between group A, group $\mathrm{B}$ and group $\mathrm{C}$ at baseline at $5 \%$ significant level $(p=<0.001)$. The mean GI score on $45^{\text {th }}$ day for Group A, Group B and Group C were $0.069 \pm 0.042,0.039 \pm 0.015$ and $0.059 \pm 0.049$ respectively. There was no statistical difference in mean GI score between group A, group B and group $C$ at 45 days at $5 \%$ significant level $(p=0.117)$ (Table 1). The GI score reduction from baseline to 45 days was found to be $0.502 \pm 0.239$ in group A, $0.826 \pm$ 0.172 in group $\mathrm{B}$ and $0.493 \pm 0.220$ in group C. One way ANOVA was used to check the GI score reduction among the groups. It showed a statistical significant difference in mean GI reduction in group A, group $\mathrm{B}$ and group $\mathrm{C}(\mathrm{p}=$ $<0.001$ ) (Table 2). A pair wise comparison was done using Scheffe post hoc test, there was a statistical significant difference in GI score reduction in group B $(\mathrm{p}<0.001)$.

The mean SBI score at baseline for Group A, Group B and Group C were $1.563 \pm 0.218,1.845 \pm 0.186$ and $1.532 \pm 0.221$ respectively. There was a statistical significant difference in mean SBI score between group A, group B \& group $C$ at baseline at $5 \%$ significant level $(p=<0.001)$. The mean score on $45^{\text {th }}$ day for Group A, Group B and Group C were $0.189 \pm 0.149,0.083 \pm 0.031$ and $0.251 \pm 0.337$ respectively. There was no statistical difference in SBI score between group A, group B and group $\mathrm{C}$ at 45 days at $5 \%$ significant level $(\mathrm{p}=0.105)$ (Table 1). The SBI score reduction from baseline to 45 days was found to be $1.375 \pm 0.190$ in group A, $1.761 \pm$ 0.195 in group B and $1.281 \pm 0.362$ in group C. One way ANOVA was used to check the SBI score reduction among the groups. It showed a statistical significant difference in mean SBI reduction in group A, group B and group $\mathrm{C}(\mathrm{p}=<0.001)$ (Table 2$)$. The pair wise comparison was done using Scheffe post hoc test, there was a statistical significant difference in SBI score reduction in group $B(p=<0.001)$.
Table 1: Comparison of Clinical parameters between groups at different visits

\begin{tabular}{|c|c|c|c|c|c|c|c|}
\hline & Visit & & $\mathbf{N}$ & Mean & SD & Fvalue* & Pvalue \\
\hline \multirow{6}{*}{ PI } & \multirow{3}{*}{ Day 1} & Group A & \multirow{3}{*}{15} & 0.915 & 0.303 & \multirow{3}{*}{3.562} & \multirow{3}{*}{$0.037^{\dagger}$} \\
\hline & & Group B & & 0.917 & 0.088 & & \\
\hline & & Group C & & 0.737 & 0.185 & & \\
\hline & \multirow{3}{*}{ Day 45} & Group A & 15 & 0.100 & 0.041 & \multirow{3}{*}{2.494} & \multirow{3}{*}{0.095} \\
\hline & & Group B & & 0.077 & 0.031 & & \\
\hline & & Group C & & 0.074 & 0.030 & & \\
\hline \multirow{6}{*}{ GI } & \multirow{3}{*}{ Day 1} & Group A & 15 & 0.571 & 0.238 & \multirow{3}{*}{9.730} & \multirow{3}{*}{$<0.001$} \\
\hline & & Group B & & 0.865 & 0.164 & & \\
\hline & & Group C & & 0.552 & 0.241 & & \\
\hline & \multirow{3}{*}{ Day 45} & Group A & 15 & 0.069 & 0.042 & \multirow{3}{*}{2.262} & \multirow{3}{*}{0.117} \\
\hline & & Group B & & 0.039 & 0.015 & & \\
\hline & & Group C & & 0.059 & 0.049 & & \\
\hline \multirow{6}{*}{ SBI } & \multirow{3}{*}{ Day 1} & Group A & 15 & 1.563 & 0.218 & \multirow{3}{*}{10.117} & \multirow{3}{*}{$<0.001$} \\
\hline & & Group B & & 1.845 & 0.186 & & \\
\hline & & Group C & & 1.532 & 0.221 & & \\
\hline & \multirow{3}{*}{ Day 45} & Group A & 15 & 0.189 & 0.149 & \multirow{3}{*}{2.377} & \multirow{3}{*}{0.105} \\
\hline & & Group B & & 0.083 & 0.031 & & \\
\hline & & Group C & & 0.251 & 0.337 & & \\
\hline
\end{tabular}

PI- Plaque index, GI-Gingival index, SBI-Sulcus Bleeding index, $\mathrm{N}$ - Number of patients $* \mathrm{~F}$ values are obtained using One way ANOVA test. $\dagger \mathrm{P}<0.05$ is considered to be statistically significant.

Table 2: Inter-group comparison of changes in clinical parameters

\begin{tabular}{|c|l|l|l|l|l|l|}
\hline & Groups & N & Mean & SD & F value* & P value \\
\hline $\begin{array}{c}\text { Change } \\
\text { in PI }\end{array}$ & Group A & & 0.815 & 0.269 & & \\
\hline & Group B & 15 & 0.839 & 0.086 & 3.527 & $0.038^{\dagger}$ \\
\hline \multirow{2}{*}{$\begin{array}{c}\text { Group C } \\
\text { in GI }\end{array}$} & $\begin{array}{lll}\text { Group A } \\
\text { Group B }\end{array}$ & 15 & 0.663 & 0.191 & & \\
\hline & Group C & & 0.826 & 0.172 & 11.900 & $<0.001$ \\
\hline \multirow{2}{*}{$\begin{array}{l}\text { Change } \\
\text { in SBI }\end{array}$} & Group A & & 1.374 & 0.190 & & \\
\hline & Group B & 15 & 1.761 & 0.195 & 14.206 & $<0.001$ \\
\hline & Group C & 1.281 & 0.362 & & \\
\hline
\end{tabular}

PI- Plaque index, GI-Gingival index, SBI-Sulcus Bleeding index, N- Number of patients, SD-Standard Deviation *F values are obtained using One way ANOVA test.

$\uparrow \mathrm{P}<0.05$ is considered to be statistically significant.

On intra- group comparison of difference in all clinical parameters at baseline and on the $45^{\text {th }}$ day, all the three groups showed a statistically significant difference $(\mathrm{P}<0.001)$ (Table 3$)$. 
Ranjini Chandraprakash et.al., Cocos nucifera, Sesamum indicum and Chlorhexidine on gingivitis

Table 3: Intra-group comparison of Clinical parameters (Paired t test)

\begin{tabular}{|c|c|c|c|c|c|c|c|}
\hline & Group & Visit & $\mathbf{N}$ & Mean & SD & *t value & $\mathbf{p}$ \\
\hline \multirow{6}{*}{ PI } & \multirow{2}{*}{ Group A } & Day 1 & \multirow{2}{*}{15} & 0.9157 & 0.30357 & \multirow{2}{*}{11.731} & \multirow{2}{*}{$<0.001^{\dagger}$} \\
\hline & & Day 45 & & 0.1006 & 0.04104 & & \\
\hline & \multirow{2}{*}{ c } & Day 1 & \multirow{2}{*}{15} & 0.9172 & 0.08816 & \multirow{2}{*}{37.720} & \multirow{2}{*}{$<0.001 \dagger$} \\
\hline & & Day 45 & & 0.0776 & 0.03196 & & \\
\hline & \multirow{2}{*}{ Group C } & Day 1 & \multirow{2}{*}{15} & 0.7379 & 0.18550 & \multirow{2}{*}{13.396} & \multirow{2}{*}{$<0.001 \dagger$} \\
\hline & & Day 45 & & 0.0747 & 0.03036 & & \\
\hline \multirow{6}{*}{ GI } & \multirow{2}{*}{ Group A } & Day 1 & \multirow{2}{*}{15} & 0.5719 & 0.23873 & \multirow{2}{*}{8.118} & \multirow{2}{*}{$<0.001 \dagger$} \\
\hline & & Day 45 & & 0.0691 & 0.04295 & & \\
\hline & \multirow{2}{*}{ Group B } & Day 1 & \multirow{2}{*}{15} & 0.8657 & 0.16449 & \multirow{2}{*}{18.574} & \multirow{2}{*}{$<0.001^{\dagger}$} \\
\hline & & Day 45 & & 0.0396 & 0.01512 & & \\
\hline & \multirow{2}{*}{ Group C } & Day 1 & \multirow{2}{*}{15} & 0.5527 & 0.24144 & \multirow{2}{*}{8.681} & \multirow{2}{*}{$<0.001^{\dagger}$} \\
\hline & & Day 45 & & 0.0593 & 0.04906 & & \\
\hline \multirow{6}{*}{ SBI } & \multirow{2}{*}{ Group A } & Day 1 & \multirow{2}{*}{15} & 1.5639 & 0.21837 & \multirow{2}{*}{27.909} & \multirow{2}{*}{$<0.001 \dagger$} \\
\hline & & Day 45 & & 0.1894 & 0.14934 & & \\
\hline & \multirow{2}{*}{ Group B } & Day 1 & \multirow{2}{*}{15} & 1.8451 & 0.18662 & \multirow{2}{*}{34.884} & \multirow{2}{*}{$<0.001^{\dagger}$} \\
\hline & & Day 45 & & 0.0832 & 0.03178 & & \\
\hline & \multirow{2}{*}{ Group C } & Day 1 & & 1.5329 & 0.22166 & & \\
\hline & & Day 45 & $1 J$ & 0.2515 & 0.33742 & $15 . / 04$ & 0.001 \\
\hline
\end{tabular}

PI-Plaque index, GI-Gingival index, SBI-Sulcus Bleeding index, N- Number of patients, SD- Standard deviation, $* t$ values - are obtained using Student paired $t$ test. ${ }^{\dagger} \mathrm{P}<0.05$ is considered to be statistically significant.

The mean IL-6 score at baseline for Group A, Group B and Group C were $1.430 \pm 0.740,0.807 \pm 0.87$ and $0.778 \pm 0.825$ respectively. There was no statistical significant difference between group A, group B and group $C$ at baseline at $5 \%$ significant level $(p=0.057)$. The mean IL-6 score at 45 days for Group A, Group B and Group C were $0.612 \pm 0.766,0.413 \pm 0.555$ and $1.066 \pm 0.862$ respectively. There was no statistical significant difference between group $\mathrm{A}$, group $\mathrm{B}$ and group $\mathrm{C}$ at 45 days at $5 \%$ significant level $(\mathrm{p}=0.056)$. The mean IL-6 score reduction at baseline was $0.817 \pm$ 1.355 in group A, $0.394 \pm 0.938$ in group B and -0.288 \pm 0.822 in group $\mathrm{C}$ (Table 4$)$.

Table 4: Comparison of Interleukin-6 between groups at different visits

\begin{tabular}{|c|c|c|c|c|c|c|}
\hline & & $\mathbf{N}$ & Mean & SD & F & $\mathbf{P}$ \\
\hline \multirow{3}{*}{ Day 1} & Group A & \multirow{3}{*}{15} & 1.430 & 0.740 & \multirow{3}{*}{3.076} & \multirow{3}{*}{0.057} \\
\hline & Group B & & 0.807 & 0.867 & & \\
\hline & Group C & & 0.778 & 0.825 & & \\
\hline \multirow{3}{*}{ Day 45} & Group A & \multirow{3}{*}{15} & 0.612 & 0.766 & \multirow{3}{*}{3.080} & \multirow{3}{*}{0.056} \\
\hline & Group B & & 0.413 & 0.555 & & \\
\hline & Group C & & 1.066 & 0.862 & & \\
\hline \multirow{3}{*}{ Difference } & Group A & \multirow{3}{*}{15} & 0.817 & 1.355 & \multirow{3}{*}{4.125} & \multirow{3}{*}{0.023} \\
\hline & Group B & & 0.394 & 0.938 & & \\
\hline & Group C & & -0.288 & 0.822 & & \\
\hline
\end{tabular}

$\mathrm{N}$ - Number of patients, SD-Standard Deviation *F values are obtained using One way ANOVA test. $\dagger$ $\mathrm{P}<0.05$ is considered to be statistically significant.
On Intra group comparison of differences, there was a statistical significant difference was seen in group $A(p=0.035)$ and no statistical significant difference seen in group $\mathrm{B}(\mathrm{P}=0.126)$ and group $\mathrm{C}(\mathrm{P}=0.196)$ (Table 5).

Table 5: Intra-group comparison of Interluekin-6 (Paired $t$ test)

\begin{tabular}{|c|c|c|c|c|c|}
\hline Group & Visit & N & Mean & SD & p \\
\hline \multirow{2}{*}{ Group A } & Day 1 & 15 & 1.430 & 0.740 & 0.035 \\
\cline { 2 - 6 } & Day 45 & 15 & 0.612 & 0.766 & \\
\hline \multirow{2}{*}{ Group B } & Day 1 & 15 & 0.807 & 0.867 & \multirow{2}{*}{0.126} \\
\cline { 2 - 6 } & Day 45 & 15 & 0.413 & 0.555 & \\
\hline \multirow{2}{*}{ Group C } & Day 1 & 15 & 0.778 & 0.825 & \multirow{2}{*}{0.196} \\
\cline { 2 - 6 } & Day 45 & 15 & 1.066 & 0.862 & \\
\hline
\end{tabular}

N- Number of patients, SD- Standard deviation, $\dagger \mathrm{P}<0.05$ is considered to be statistically significant.

\section{Discussion}

Dental plaque plays a vital role in causation and progression of various conditions affecting the oral cavity. Therefore, plaque control measures play a pivotal role in treating and containing these conditions. Various antimicrobial formulations in the form of mouth rinses have been used as an adjunct to mechanical means of plaque control. Among these, chlorhexidine has been considered a gold standard against which other antiplaque agents are compared for their efficacy. Disadvantages of chlorhexidine has prevented its longterm use. This study was designed to evaluate the effect of Cocos nucifera and Sesamum indicum mouthwash on gingivitis in comparison to chlorhexidine.

The ancient system of Indian medicine, Ayurveda advocates the use of preparations derived from plants and trees, to effectively treat various pathologic conditions (10). Herbal products being natural, show lesser side effects and are economical compared to their synthetic counterparts. Cocos nucifera (Coconut) is most routinely used as a component of food worldwide. At concentrations of 5\% to $40 \%(\mathrm{w} / \mathrm{w})$ coconut oil has shown to have bactericidal activity (11). Taheri JB et al., in an in-vitro study have shown that sucrose monolaurate, present in coconut reduces sucrose oxidation and glycolysis caused by Streptococcus mutans thus could possess anti-caries effect (12). Coconut proteins when fed to immunosuppressed animals, showed marked increase in levels of hemoglobin, red blood cells, neutrophils, monoctyes, B and T-Lymphocytes suggesting immunomodulatory activity (13). Peedikayi FC et al., compared the effect of coconut oil and chlorhexidine on Streptococcus mutans. In this in vivo study they concluded that Coconut oil is as effective as chlorhexidine in the reduction of Streptococcus mutans (14).

Sesame oil is one of the common ingredients in Indian food. In our study, sesame oil mouth wash group was equally effective in reducing gingival inflammation in comparison with chlorhexidine mouthwash. Similar results were reported by Asokan et al., who compared 
Sesame oil pulling with chlorhexidine mouth in the treatment of plaque-induced gingivitis, showing statistically significant reduction of Streptococcus mutans count in plaque. Their study also found sesame oil equally effective to chlorhexidine in reducing gingivitis (15).

The results of our study are in concurrence with that of Kaliamoorthy $\mathrm{S}$ et al., in which both coconut oil and sesame oil were effective in reducing severity of gingivitis (16).

Interleukin-6 in considered having proinflammatory activity in relation the periodontal disease progression (17). To the best of our knowledge, no studies are found in the literature reporting the effect of Cocos nucifera, Sesame oil on IL-6 levels in the treatment of plaque induced gingivitis. In our study, on intra group comparison of differences, there was a statistical significant difference was seen in Cocos nucifera group $(\mathrm{p}=0.035)$ and no statistical significant difference seen in Sesame oil or chlorhexidine groups. This could suggest anti-inflammatory property of Cocos nucifera.

The results of our study, showed statistically significant reduction in GI, PI, SBI scores and IL-6 levels in all three groups on the $45^{\text {th }}$ day, suggesting both could effectively reduce gingivitis. Small sample size and short duration were the limitations of our study. However, long term studies with large sample size would be necessary to establish the results of our study.

\section{Conclusion}

In the present study, Cocos nucifera and Sesame indicum mouthwashes in comparison with chlorhexidine, effectively reduced plaque and gingivitis. Being herbal products and those used routinely in food, both Cocos nucifera and Sesame indicum could be used as an economical and natural substitute to synthetic plaque control agents.

\section{References}

1. Nazir MA. Prevalence of periodontal disease, its association with systemic diseases and prevention. Int J Health Sci 2017;11:72-80.

2. Jones CG. Chlorhexidine: is it still the gold standard?. Periodontol 2000 1997;15:55-62.

3. James P, Worthington HV, Parnell C, Harding M, Lamont $\mathrm{T}$, Cheung $\mathrm{A}$, et al. Chlorhexidine mouthrinse as an adjunctive treatment for gingival health. Cochrane Database of Sys Rev 2017;3:CD008676.

4. DebMandal M, Mandal S. Coconut (Cocos nucifera L.: Arecaceae): In health promotion and disease prevention. Asian Pac J Trop Med 2011;4:241-7.
5. Barnabe W, de Mendonça Neto T, Pimenta FC, Pegoraro LF, Scolaro JM. Efficacy of sodium hypochlorite and coconut soap used as disinfecting agents in the reduction of denture stomatitis, Streptococcus mutans and Candida albicans. J Oral Rehabil 2004;31:453-9.

6. Asokan S. Oil pulling therapy. Indian J Dent Res 2008;19:169.

7. Silness J, Loe H. Periodontal disease in pregnancy II, correlation between oral hygiene and periodontal condition. Acta Odontol Scand 1964;4:487-95.

8. Loe H, Silness J. Periodontal disease in pregnancy I, Prevalence and severity. Acta Odontol Scand 1963;21:533-51.

9. Muhlemann HR, Son S. Gingival bleeding a leading symptom in initial gingivitis. Helvetica Odontologica Acta 1971;15:107-13.

10. Pandita V, Patthi B, Singla A, Singh S, Malhi R, Vashishtha V. Dentistry meets nature-role of herbs in periodontal care: A systematic review. J Indian Assoc Public Health Dent 2014;12:148-56

11. Oyi AR, Onaolapo JA, Obi RC. Formulation and antimicrobial studies of coconut (Cocos nucifera Linne) Oil. Res J Appl S Eng Tech 2010;2:133-7.

12. Taheri JB, Espineli FW, Lu H, Asayesh M, Bakshi M, Nakhostin MR, et al. Antimicrobial effect of coconut flour on oral microflora: An in vitro study. Res J Biol Sci 2010;5:456-9.

13. Vigila AG, Baskaran X. Immunomodulatory effect of coconut protein on cyclophosphamide induced immune suppressed Swiss Albino mice. Ethnobot Leaflets 2008;12:1206-12.

14. Peedikayil FC, Remy V, John S, Chandru TP, Sreenivasan P, Bijapur GA. Comparison of antibacterial efficacy of coconut oil and chlorhexidine on Streptococcus mutans: An in vivo study. J Int Soc Prevent Communit Dent 2016;6:447-52.

15. Asokan S, Emmadi P, Chamundeswari R. Effect of oil pulling on plaque induced gingivitis: A randomized, controlled, triple-blind study. Indian J Dent Res 2009;20:47-51.

16. Kaliamoorthy S, Pazhani A, Nagarajan $M$, Meyyappan A, Rayar S, Mathivanan S. Comparing the effect of coconut oil pulling practice with oil pulling using sesame oil in plaque-induced gingivitis: A prospective comparative interventional study. J Nat Sc Biol Med 2018;9:165-8.

17. Ross JH, Hardy DC, Schuyler CA, Slate EH, Mize TW, Huang Y. Expression of periodontal interleukin-6 protein is increased across patients with neither periodontal disease nor diabetes, patients with periodontal disease alone and patients with both diseases. J Periodontal Res 2010;45:688-94. 